\title{
Spin transport in antiferromagnetic insulators: progress and challenges
}

\author{
Dazhi Hou (i) ${ }^{1,2}$, Zhiyong Qiu ${ }^{3,4}$ and Eiji Saitoh ${ }^{2,5,6}$
}

\begin{abstract}
Spin transport is a key process in the operation of spin-based devices that has been the focus of spintronics research for the last two decades. Conductive materials, such as semiconductors and metals, in which the spin transport relies on electron diffusion, have been employed as the channels for spin transport in most studies. Due to the absence of conduction electrons, the potential to be a spin channel has long been neglected for insulators. However, since the demonstration of spin transmission through a ferromagnetic insulator, it was realized that insulators with magnetic ordering can also serve as channels for spin transport. Here, the recent progress of spin transport in antiferromagnetic insulators is briefly described with an introduction to the experimental techniques. The observations regarding the temperature dependence of spin transmission, spin current switching and the negative spin Hall magnetoresistance are discussed. We also include the challenges for developing the functionality of antiferromagnetic insulators as well as the unresolved problems from the experimental observations.
\end{abstract}

A core mission for spintronics is to develop spin-based devices in which information is processed by spin rather than charge ${ }^{1}$. Efficient spin transport is a prerequisite for the operation of spin-based devices. If spin transport can be further controlled by some external parameters, e.g., gate voltage, a spin transistor can be realized ${ }^{2}$. Following these visions from the pioneers, spin transport has been studied extensively in various materials, and different approaches have been developed for its manipulation ${ }^{3}$.

It is likely that the mainstream materials for spin transport have been semiconductors and metals in which the spin current is essentially electron diffusion with a difference between the up-spin and down-spin electron chemical potentials because the spin current was initially introduced and demonstrated with conduction

\footnotetext{
Correspondence: Dazhi Hou (dazhi.hou@foxmail.com)

${ }^{1}$ International Centre for Quantum Design of Functional Materials (ICQD), Hefei National Laboratory for Physical Sciences at the Microscale (HFNL), and Synergetic Innovation Center of Quantum Information and Quantum Physics, University of Science and Technology of China, 230026 Hefei, China

${ }^{2}$ WPI Advanced Institute for Materials Research, Tohoku University, Sendai 9808577, Japan

Full list of author information is available at the end of the article.

These authors contributed equally: Dazhi Hou, Zhiyong Qiu
}

electrons $^{4,5}$. However, since a spin current can generally be considered as a flow of angular momentum, electrons should not be the only choice for the carrier of spin. In principle, systems comprising any particle or quasiparticle with an angular momentum degree of freedom can host spin current. For instance, the spin transport mediated by magnons in $\mathrm{Y}_{3} \mathrm{Fe}_{5} \mathrm{O}_{12}$ (YIG), a ferrimagnetic insulator, was demonstrated by Kajiwara et al. ${ }^{6}$. From then on, the study of spin transport in ferromagnetic insulators has been a topic of interest in the spintronics field.

Since the spin current in ferromagnetic insulators is believed to be mediated by magnons, antiferromagnetic insulators (AFMI), which also host magnons, hold potential for spin transport as well. Indeed, the demonstration of spin transport through antiferromagnetic insulators was first achieved by Wang et al. $^{7}$, which was followed by similar results from other groups ${ }^{8,9}$. This breakthrough included a new class of materials for spin transport, which opened up new possibilities for future device development.

Here, the experimental configurations for the study of spin transport in AFMI are briefly introduced with a discussion of the observation results. Figure 1 illustrates

\section{(c) The Author(s) 2019}

(c) (i) Open Access This article is licensed under a Creative Commons Attribution 4.0 International License, which permits use, sharing, adaptation, distribution and reproduction cc) in any medium or format, as long as you give appropriate credit to the original author(s) and the source, provide a link to the Creative Commons license, and indicate if changes were made. The images or other third party material in this article are included in the article's Creative Commons license, unless indicated otherwise in a credit line to the material. If material is not included in the article's Creative Commons license and your intended use is not permitted by statutory regulation or exceeds the permitted use, you will need to obtain permission directly from the copyright holder. To view a copy of this license, visit http://creativecommons.org/licenses/by/4.0/. 


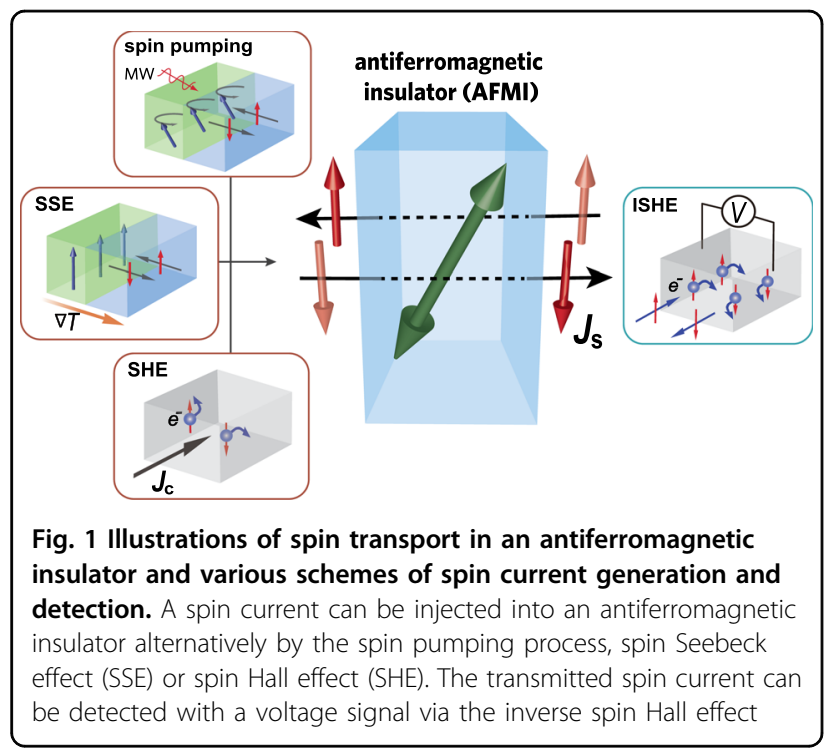

the essential processes for the observation of spin transport in AFMI from left to right: spin current generation, spin transmission, and spin current detection. The spin pumping process, spin Seebeck effect or spin Hall effect can be used to generate and inject a spin current into an AFMI, in which the driving forces are microwave, temperature gradient and charge current, respectively ${ }^{10-12}$. The spin current transmitted through an AFMI can be detected by a voltage in an adjacent heavy metal via the inverse spin Hall effect ${ }^{10}$.

The sample structure used in the ref. ${ }^{7}$, a $\mathrm{Pt} / \mathrm{NiO} / \mathrm{YIG}$ trilayer, is taken as an example and illustrated in Fig. 2a. A spin current is injected into $\mathrm{NiO}$, which is an antiferromagnetic insulator (AFMI), from $\mathrm{Y}_{3} \mathrm{Fe}_{5} \mathrm{O}_{12}$ by spin pumping and detected by the inverse spin Hall effect (ISHE) in $\mathrm{Pt}^{10}$. Surprisingly, the ISHE voltage in the Pt/ $\mathrm{NiO} / \mathrm{YIG}$ device is even larger than that in the Pt/YIG device when the $\mathrm{NiO}$ is approximately $1 \mathrm{~nm}$, which means that the spin current is somehow enhanced by the presence of the $\mathrm{NiO}$ interlayer. Such a counterintuitive result was also found in the spin Seebeck measurement for Pt/ $\mathrm{NiO} / \mathrm{YIG}^{13}$, which is shown in Fig. 2e. An instant question following these studies is: what is the optimal condition for spin transport in an AFMI? It is important both for the potential of AFMIs in further applications and for an understanding of the microscopic mechanism of spin transport. The temperature dependence of the damping constant in a permalloy/Cu/IrMn/Al device initially shed light on this issue, in which IrMn is an antiferromagnetic alloy. These results show that the spin current injection by spin pumping has an anomalous enhancement at a temperature that increases with the IrMn thickness, as shown in Fig. $2 \mathrm{f}^{14}$. Although it is quite challenging to determine the ordering temperature of the IrMn film in their devices, which is below $1.5 \mathrm{~nm}$, it was argued that the spin pumping enhancement temperature may be close to the Néel temperature.

Although the IrMn alloy is metallic rather than insulating, the result undoubtedly highlights the intriguing behavior of spin transport in the phase transition regime of antiferromagnets.

Shortly after the publication of the spin pumping result for $\mathrm{IrMn}^{14}$, spin transmission was reported in a $\mathrm{Pt} / \mathrm{CoO} /$ YIG device by a temperature-dependent spin pumping inverse spin Hall measurement and directly determined the Néel point of the $\mathrm{CoO}$ by $\mathrm{X}$-ray linear dichroism (XMLD) at the same time ${ }^{15}$. Figure $2 b$ plots the representative ISHE voltage $\left(V_{\text {ISHE }}\right)$ measured with a magnetic field that was swept at different temperatures, and Fig. 2c is the temperature dependence of the $V_{\text {ISHE}}$, which shows a peak at approximately $200 \mathrm{~K}$. Figure $2 \mathrm{~d}$ is the result of the XMLD measurement from the same structure, which yields a Néel temperature of $210 \mathrm{~K}$ with an error bar of $10 \mathrm{~K}$. Thus, the spin current transmission in $\mathrm{CoO}$ does have an enhancement around the Néel temperature, which is consistent with the result in ${ }^{14}$. Similar temperature dependence was also observed by the spin Seebeck measurement in similar structures ${ }^{13}$.

All of these results strongly suggest that the thermal magnon population is important for spin transport in AFMI. Nevertheless, since spin fluctuation cannot be neglected in the vicinity of the Néel temperature, it is still a theoretical issue whether spins are transported in the form of a magnon or a spin fluctuation. On the other hand, a key experiment that could determine whether the transmitted spin current is coherent is still not available ${ }^{16}$. The evanescent mode is argued to cause the amplification of spin current in $\mathrm{NiO}$, but it seems hard to explain the vanishing spin transmission in AFMIs at low temperatures $^{7,17}$. Further progress on the spin transport mechanism determination may be made by the temperature dependence of the $\mathrm{AC} \mathrm{ISHE}^{18,19}$, from which the coherence of the AC spin current can be checked.

So far, the studies mentioned above were more about the characterization of the spin transport property in AFMIs $^{7-9,13-15}$, while the control of the spin transport in the AFMIs, e.g., the switching of a spin current, which is indispensable for next-step applications, has not been achieved yet. Recently, isothermal switching of a spin current was demonstrated in $\mathrm{Cr}_{2} \mathrm{O}_{3}$, in which the spin transmission modulation is greater than $500 \%$ under a magnetic field ${ }^{20}$. Figure 3 a shows the illustrations for the experimental configuration, and Fig. $3 \mathrm{~b}$ is the crosssection TEM image of the device. Here, the $\mathrm{YIG} / \mathrm{Cr}_{2} \mathrm{O}_{3} / \mathrm{Pt}$ structure is employed, in which a spin current is driven by a temperature gradient, $\nabla T$, from the YIG into the $\mathrm{Cr}_{2} \mathrm{O}_{3}$ by the spin Seebeck effect ${ }^{11,21,22}$. In contrast to the samples used in a previous study, the $\mathrm{Cr}_{2} \mathrm{O}_{3}$ layer has a wellaligned out-of-plane Néel vector due to its single 

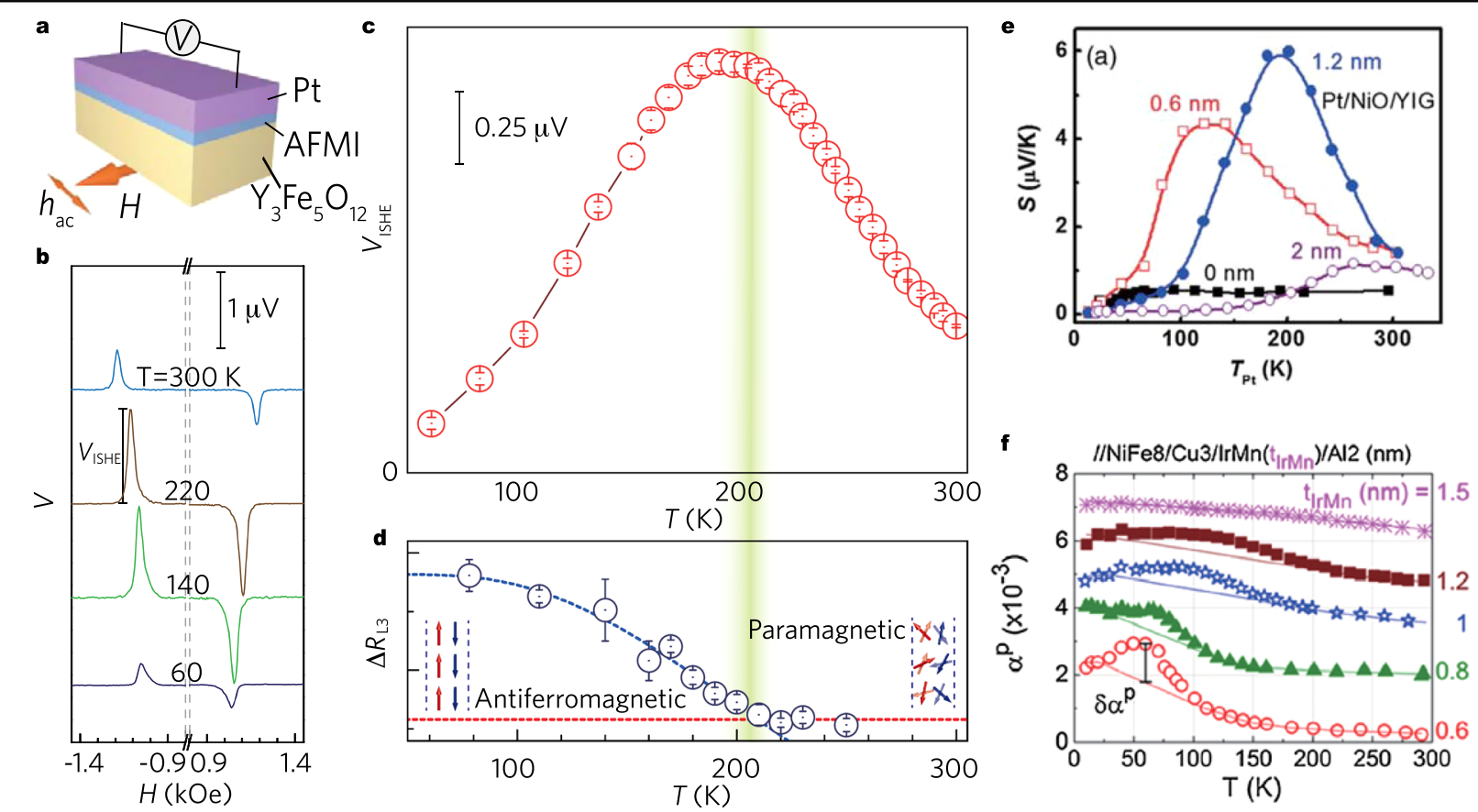

Fig. 2 a A schematic illustration of the device structure for spin transport in an antiferromagnetic insulator (AFMI), e.g., NiO or CoO. The ferrimagnetic insulator $\mathrm{Y}_{3} \mathrm{Fe}_{5} \mathrm{O}_{12}$ serves as the spin injector, and the transmitted spin current is detected via the inverse spin Hall effect in Pt. b The inverse spin Hall voltage observed in a magnetic field scan at various temperatures in a Pt/CoO $(6 \mathrm{~nm}) / \mathrm{YIG}$ device. c Temperature dependence of the inverse spin Hall voltage in Pt/CoO $(6 \mathrm{~nm}) / \mathrm{YIG}$. $\mathbf{d}$ Temperature dependence of the X-ray linear dichroism signal $\Delta R_{L 3}$ in Pt/CoO $(6 \mathrm{~nm}) / Y I G$, from which the Néel temperature is determined. e The spin Seebeck thermal power in a series of Pt/NiO/YIG samples with different NiO thicknesses. $\mathbf{f}$ The temperature dependence of the damping constant of permalloy/Cu/lrMn/Al samples after background subtraction for different IrMn thicknesses. e Adapted from the ref. ${ }^{13} . \mathbf{f}$ Adapted from the ref. ${ }^{14}$

crystalline structure and uniaxial magnetic anisotropy. Figure $3 \mathrm{c}$ plots the temperature dependence of the spin Seebeck voltage in a YIG/ $\mathrm{Cr}_{2} \mathrm{O}_{3} / \mathrm{Pt}$ device measured with an in-plane magnetic field, which shows a sudden transition from the spin conductor state to the spin nonconductor state within $14 \mathrm{~K}$ of the Néel temperature. Such behavior is in sharp contrast to the gradual decay of spin transmission below the Néel temperature in $\mathrm{CoO}$ and $\mathrm{NiO}^{13,15}$. The suppression of spin transmission in the antiferromagnetic phase can be understood by the symmetry requirement of the magnon spin current: the spin polarization of magnons must be parallel to the Néel vector, which is different from the arbitrary spin polarization direction in electron systems. This property can be inferred from the spin transmission in the YIG, in which the spin current is blocked when the magnetization of YIG is perpendicular to the direction of the injected spin from Pt. Thus, the configuration in Fig. 3a corresponds to an "OFF" state for spin transmission due to the orthogonal relative orientation between the magnetization of YIG and the Néel vector of $\mathrm{Cr}_{2} \mathrm{O}_{3}$.

Then, it is highly desirable to reach an "ON" state for spin transmission in the same device. Since the inverse spin Hall effect in Pt can only detect the spin with an in- plane orientation, a nonzero component of the Néel vector in $\mathrm{Cr}_{2} \mathrm{O}_{3}$ in the sample plane is essential for measurable spin transmission for the present device. From the calculation of the Néel vector orientation in a uniaxial antiferromagnet under an external magnetic field, we found that the N'eel vector can be tilted slightly when the external field is neither parallel nor perpendicular to $\mathrm{it}^{23}$. Guided by this understanding, we measured the temperature dependence of the spin Seebeck effect in the $\mathrm{Pt} / \mathrm{Cr}_{2} \mathrm{O}_{3} / \mathrm{YIG}$ device under different magnetic fields with a 20-degree tilting angle relative to the sample normal, the results of which are plotted in Fig. 3d. With an increase in the field magnitude from $0.5 \mathrm{~T}$ to $2.5 \mathrm{~T}$, the enhancement of the spin Seebeck voltage is observed due to the rotation of the Nél vector. The change ratio due to the magnetic field, Ratio $\left(T_{\mathrm{s}}\right)_{@ \mathrm{H}}=\left(V_{\mathrm{SSE} @ \mathrm{H}}-V_{\mathrm{SSE} @ 0.5 \mathrm{~T}}\right) / V_{\mathrm{SSE} @ 0.5 \mathrm{~T}}$, is plotted in Fig. 3e, which exceeds 500\% for the temperature regime just below the Néel point and demonstrates an "ON" state for spin transmission. A systematic field angle dependence of the $V_{\mathrm{SSE}}$ results can be found in the original paper, which supports the Néel vector rotation scenario $^{20}$. The Néel vector direction-dependent spin transport in AFMI has also been reported in a Pt/hematite/Pt lateral structure ${ }^{24}$. 


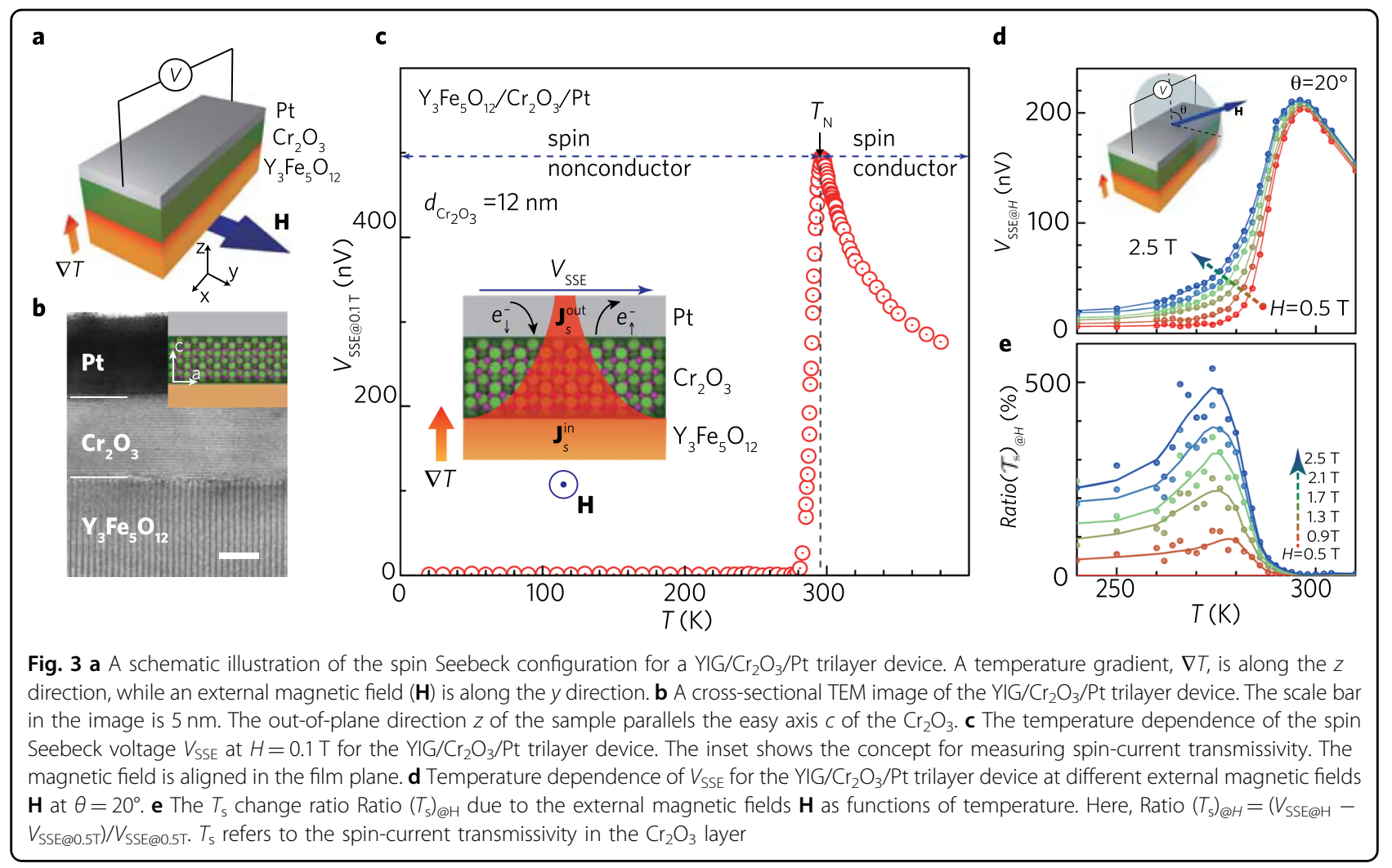

In addition to the recently studied spin transport, antiferromagnetic insulators also show a nontrivial effect on the magnetoresistance in neighboring heavy metals, such as Pt. In 2016, Shang et al. reported the temperature dependence of the magnetoresistance measurement in a $\mathrm{Pt} / \mathrm{NiO} / \mathrm{YIG}$ structure ${ }^{25}$. It was found that $\mathrm{Pt}$ shows a typical spin Hall magnetoresistance (SMR) behavior at room temperature, which was described for a Pt/YIG bilayer structure ${ }^{26,27}$. However, it is surprising that the SMR in Pt has a sign change for $T<70 \mathrm{~K}$, which is hard to understand with the standard SMR model. Since the effect of $\mathrm{NiO}$ has been shown to quantitatively modulate the spin transmission between YIG and Pt, it is unexpected that the SMR shows a negative sign at low temperatures. We reproduced a sign change in a $\mathrm{Pt} / \mathrm{NiO} / \mathrm{YIG}$ device, and similar results were reported by another group ${ }^{28}$. The measurement configurations are illustrated in Fig. $4 \mathrm{a}-\mathrm{c}$, and the SMR results at $260 \mathrm{~K}$ and $20 \mathrm{~K}$ are plotted in Fig. $4 \mathrm{~d}$, e, respectively, which show the same SMR symmetry with opposite signs. Thus, the task is to explain the sign change. To achieve this, the magnetoresistance is measured for the $\mathrm{Pt} / \mathrm{NiO} / \mathrm{YIG}$ samples in a wide range of $\mathrm{NiO}$ thicknesses from $1.6 \mathrm{~nm}$ to $30 \mathrm{~nm}^{29}$. We noticed that the negative SMR is still finite even when the spin transmission between Pt and YIG is completely blocked for low temperature limits and thick $\mathrm{NiO}$, indicating that the negative SMR is not caused by the spin current reflected from the YIG. Based on this observation, we develop the following interpretation, as illustrated in Fig. 3f. We attribute the negative SMR at low temperature to the $\mathrm{NiO}$, which is assumed to have a $90^{\circ}$ coupling with the YIG, which is a so-called spin-flop coupling ${ }^{30}$. In other words, the $\mathrm{Pt} / \mathrm{NiO}$ interface contributes a negative SMR because the Néel vector of $\mathrm{NiO}$ is always perpendicular to the magnetic field. It is worth noting that $\mathrm{NiO}$ shows parallel coupling with a ferromagnet in some cases and winds up in a domain under manipulation ${ }^{31,32}$. With increasing temperature, the spin transmission between the Pt and YIG through the $\mathrm{NiO}$, which contributes the conventional positive SMR, is enhanced. Therefore, a sign change of the SMR occurs when these two contributions compensate for each other. Recently, the spin-flop coupling between the NiO and YIG was confirmed by Luan et al. by polarized neutron reflectometry ${ }^{33}$.

The negative SMR in the $\mathrm{Pt} / \mathrm{NiO}$ bilayer was also reported by several other groups ${ }^{34,35}$. These works open the possibility of using AFM insulators as memory materials since orthogonal orientations of the Néel vector can be electrically determined. The prototype memory devices based on $\mathrm{Pt} / \mathrm{NiO}$ bilayer structures were recently demonstrated independently by two groups, the results of which are shown in Fig. 4g, $\mathrm{h}^{36,37}$.

Finally, we would like to discuss the challenges and unresolved problems in the study of spin transport in 


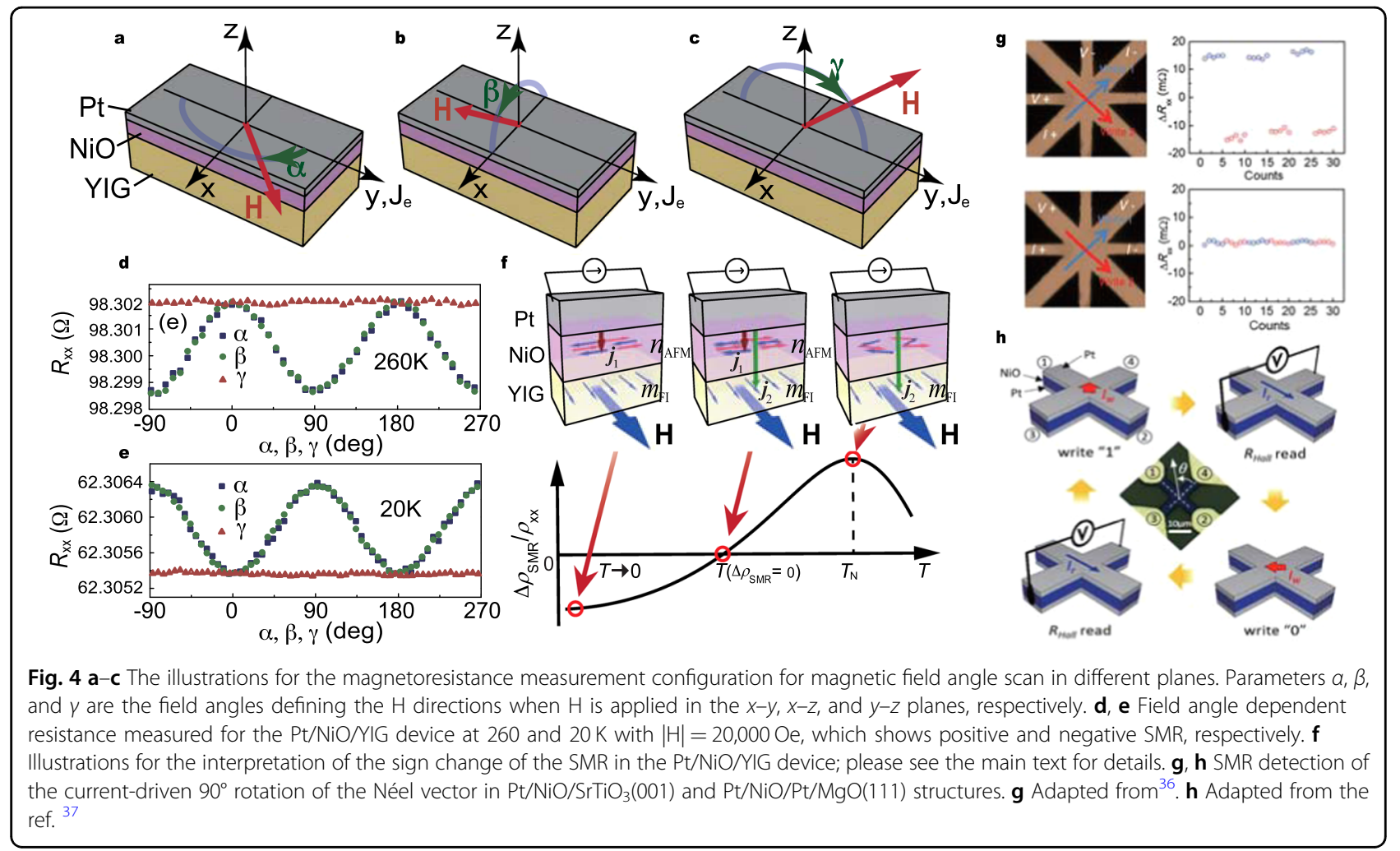

AFMIs. Although spin transport has been demonstrated in several AFMIs, the mechanisms of spin transfer through an AFMI, which is indispensable for further device design and development, have not been clarified. The scaling law of magnon spin current is another important issue to be addressed, which is closely related to the mechanism of spin transport. It might be approached by either a good theory capable of fitting the experimental data or a nicely designed experiment yielding the scaling law. Some unique features have been systematically captured by experiments but are still hard to understand, e.g., the pronounced microwave frequency dependence of spin pumping efficiency near the Néel point ${ }^{15}$. Meanwhile, the proposed spin transport mechanisms need to be carefully verified by some intelligent and well-controlled experiments ${ }^{16,38}$. Another challenge lies in excitation of the THz dynamics of an AFMI by a spin current, which is quite interesting but still challenging due to the large anisotropy of antiferromagnets ${ }^{39-41}$.

\section{Acknowledgements}

This work was supported by JST-ERATO "Spin Quantum Rectification", JSTPRESTO "Phase Interfaces for Highly Efficient Energy Utilization", Grant-in-Aid for Scientific Research on Innovative Area, "Nano Spin Conversion Science" (26103005 and 26103006), Grant- in-Aid for Scientific Research (S) (25220910), Grant-in-Aid for Scientific Research (A) (25247056 and 15H02012), Grant-in-Aid for Challenging Exploratory Research (26600067), Grant-in-Aid for Research Activity Start-up (25889003), and World Premier International Research Center Initiative (WPI), all from MEXT, Japan. Z.Q. acknowledges support from the "Fundamental Research Funds for the Central Universities (DUT17RC(3)073)".

\section{Author details}

'International Centre for Quantum Design of Functional Materials (ICQD), Hefei National Laboratory for Physical Sciences at the Microscale (HFNL), and Synergetic Innovation Center of Quantum Information and Quantum Physics, University of Science and Technology of China, 230026 Hefei, China. ${ }^{2}$ WPI Advanced Institute for Materials Research, Tohoku University, Sendai 980-8577, Japan. ${ }^{3}$ School of Materials Science and Engineering, Dalian University of Technology, 116024 Dalian, China. ${ }^{4}$ Institute for Materials Research, Tohoku University, Sendai 980-8577, Japan. ${ }^{5}$ Department of Applied Physics, The University of Tokyo, Hongo, Bunkyo-ku, Tokyo 113-8656, Japan. ' Advanced Science Research Center, Japan Atomic Energy Agency, Tokai 319-1195, Japan

Conflict of interest

The authors declare that they have no conflict of interest.

\section{Publisher's note}

Springer Nature remains neutral with regard to jurisdictional claims in published maps and institutional affiliations.

Received: 21 September 2018 Revised: 15 February 2019 Accepted: 14 April 2019.

Published online: 12 July 2019

\section{References}

1. Maekawa, S. Concept in Spin Electronics (Oxford University Press, Oxford, 2006).

2. Datta, S. \& Das, B. Electronic analog of the electro-optic modulator. Appl. Phys. Lett. 56, 665-667 (1990).

3. Žuti'c, I. \& Das Sarma, S. Spintronics: fundamentals and applications. Rev. Mod. Phys. 76, 323-410 (2004).

4. Johnson, M. \& Silsbee, R. H. Interfacial charge-spin coupling: injection and detection of spin magnetization in metals. Phys. Rev. Lett. 55, 1790-1793 (1985). 
5. Johnson, M. \& Silsbee, R. H. Thermodynamic analysis of interfacial transport and of the thermomagnetoelectric system. Phys. Rev. B 35, 4959-4972 (1987)

6. Kajiwara, Y. et al. Transmission of electrical signals by spin-wave interconversion in a magnetic insulator. Nature 464, 262 (2010).

7. Wang, H., Du, C., Hammel, P. C. \& Yang, F. Antiferromagnonic spin transport from Y3Fe5O12 into NiO. Phys. Rev. Lett. 113, 97202 (2014).

8. Hahn, C. et al. Conduction of spin currents through insulating antiferromagnetic oxides. EPL 108, 57005 (2014).

9. Moriyama, T. et al. Anti-damping spin transfer torque through epitaxial nickel oxide. Appl. Phys. Lett. 106, 162406 (2015).

10. Saitoh, E., Ueda, M., Miyajima, H. \& Tatara, G. Conversion of spin current into charge current at room temperature: inverse spin-Hall effect. Appl. Phys. Lett. 88, 182509 (2006).

11. Uchida, K. et al. Spin seebeck insulator. Nat. Mater. 9, 894-897 (2010).

12. Hirsch, J. E. Spin hall effect. Phys. Rev. Lett. 83, 1834-1837 (1999).

13. Lin, W., Chen, K., Zhang, S. \& Chien, C. L. Enhancement of thermally injected spin current through an antiferromagnetic insulator. Phys. Rev. Lett. 116, 186601 (2016)

14. Frangou, L. et al. Enhanced spin pumping efficiency in antiferromagnetic irmn thin films around the magnetic phase transition. Phys. Rev. Lett. 116, 077203 (2016).

15. Qiu, Z. et al. Spin-current probe for phase transition in an insulator. Nat. Commun. 7, 12670 (2016).

16. Takei, S., Moriyama, T., Ono, T. \& Tserkovnyak, Y. Antiferromagnet-mediated spin transfer between a metal and a ferromagnet. Phys. Rev. B 92, 20409 (2015).

17. Khymyn, R., Lisenkov, I., Tiberkevich, V. S., Slavin, A. N. \& Ivanov, B. A. Transformation of spin current by antiferromagnetic insulators. Phys. Rev. B 93, 224421 (2016)

18. Hahn, C. et al. Detection of microwave spin pumping using the inverse spin hall effect. Phys. Rev. Lett. 111, 217204 (2013).

19. Wei, D., Obstbaum, M., Ribow, M., Back, C. H. \& Woltersdorf, G. Spin Hall voltages from a.c. and d.c. spin currents. Nat. Commun. 5, 3768 (2014).

20. Qiu, Z. et al. Spin colossal magnetoresistance in an antiferromagnetic insulator. Nat. Mater. 17, 577-580 (2018).

21. Uchida, K. et al. Longitudinal spin Seebeck effect: from fundamentals to applications. J. Phys. Condens. Matter 26, 343202 (2014).

22. Hou, D. et al. Observation of temperature-gradient-induced magnetization. Nat. Commun. 7, 12265 (2016).

23. Wang, $\mathrm{H}$. et al. Antiferromagnetic anisotropy determination by spin Hall magnetoresistance. J. Appl. Phys. 122, 83907 (2017).
24. Lebrun, R. et al. Tunable long-distance spin transport in a crystalline antiferromagnetic iron oxide. Nature 561, 222-225 (2018).

25. Shang, T. et al. Effect of nio inserted layer on spin-hall magnetoresistance in pt/nio/yig heterostructures. Appl. Phys. Lett. 109, 032410 (2016).

26. Nakayama, H. et al. Spin hall magnetoresistance induced by a nonequilibrium proximity effect. Phys. Rev. Lett. 110, 206601 (2013).

27. Chen, Y.-T. et al. Theory of spin hall magnetoresistance. Phys. Rev. B 87, 144411 (2013).

28. Lin, W. \& Chien, C. L. Electrical detection of spin backflow from an antiferromagnetic insulator $/ \mathrm{y}_{3} \mathrm{fe}_{5} \mathrm{O}_{12}$ interface. Phys. Rev. Lett. 118, 067202 (2017).

29. Hou, D. et al. Tunable sign change of spin hall magnetoresistance in $\mathrm{Pt} / \mathrm{NiO} /$ YIG structures. Phys. Rev. Lett. 118, 147202 (2017).

30. Koon, N. C. Calculations of exchange bias in thin films with ferromagnetic/ antiferromagnetic interfaces. Phys. Rev. Lett. 78, 4865-4868 (1997).

31. Krug, I. P. et al. Impact of interface orientation on magnetic coupling in highly ordered systems: a case study of the low-indexed $\mathrm{fe}_{3} \mathrm{O}_{4} \mathrm{NiO}$ interfaces. Phys. Rev. B 78, 064427 (2008).

32. Li, J. et al. Chirality switching and winding or unwinding of the antiferromagnetic nio domain walls in $\mathrm{Fe} / \mathrm{NiO} / \mathrm{Fe} / \mathrm{CoO} / \mathrm{Ag}(001)$. Phys. Rev. Lett. 113, 147207 (2014)

33. Luan, Z. Z. et al. Interfacial coupling and negative spin hall magnetoresistance in pt/nio/yig. Appl. Phys. Lett. 113, 072406 (2018).

34. Hoogeboom, G. R., Aqeel, A., Kuschel, T., Palstra, T. T. M. \& van Wees, B. J. Negative spin Hall magnetoresistance of Pt on the bulk easy-plane antiferromagnet NiO. Appl. Phys. Lett. 111, 52409 (2017).

35. Fischer, J. et al. Spin Hall magnetoresistance in antiferromagnet/heavy-metal heterostructures. Phys. Rev. B 97, 14417 (2018).

36. Moriyama, T., Oda, K., Ohkochi, T., Kimata, M. \& Ono, T. Spin torque control of antiferro- magnetic moments in NiO. Sci. Rep. 8, 1-6 (2018). 1708.07682.

37. Chen, X. Z. et al. Antidamping-torque-induced switching in biaxial antiferromagnetic insula- tors. Phys. Rev. Lett. 120, 207204 (2018).

38. Rezende, S. M., Rodr'iguez-Su'arez, R. L. \& Azevedo, A. Diffusive magnonic spin transport in antiferromagnetic insulators. Phys. Rev. B 93, 054412 (2016).

39. Cheng, R., Xiao, D. \& Brataas, A. Terahertz antiferromagnetic spin hall nanooscillator. Phys. Rev. Lett. 116, 207603 (2016)

40. Sulymenko, O. R. et al. Terahertz-frequency spin hall auto-oscillator based on a canted anti- ferromagnet. Phys. Rev. Appl. 8, 064007 (2017).

41. Sulymenko, O. R., Prokopenko, O. V., Tyberkevych, V. S. \& Slavin, A. N. Terahertzfrequency signal source based on an antiferromagnetic tunnel junction. IEEE Magn. Lett. 9, 1-5 (2018). 